\title{
Existence of equilibria in a decentralized two-level supply chain
}

\author{
Dolores Romero Morales * and Dries Vermeulen ${ }^{\dagger}$
}

June 19, 2008

\begin{abstract}
In this paper we analyze equilibria in competitive environments under constraints across players' strategies. This means that the action taken by one player limits the possible choices of the other players. In this context the usual approach to show existence of equilibrium, Kakutani's Fixed Point theorem, cannot be applied directly. In particular, best replies against a given strategy profile may not be feasible. We devise a new fixed point correspondence to deal with the feasibility issue.

Our main motivation to study this problem of co-dependency comes from the field of supply chain planning. A set of buyers is faced with external demand over a planning horizon, and to satisfy this demand they request inputs from a set of suppliers. Both suppliers and buyers face production capacities and they plan their own production in a decentralized manner. A well-known coordination scheme for this setting is the upstream approach where the plan of the buyers is used to decide the request to the suppliers. We show the existence of equilibria for a (shared) inventory cost minimization version of this coordination scheme in which a distribution center manages the inventory of the inputs. However, we illustrate with an example that the centralized solution is not, in general, an equilibrium, suggesting that regulation may be needed.

Keywords: Equilibria, Supply Chain Coordination, Decentralization, Upstream Planning
\end{abstract}

\section{Introduction}

In a non-cooperative game we are given a set of players and their corresponding strategy sets. Players simultaneously choose their strategies, and the payoff of each player depends on the strategies chosen. Because of this structure of the payoffs, most of the literature devoted to non-cooperative games assumes that the feasible strategies of each player are independent of the strategies chosen by the other players. However, there are many relevant situations where constraints exist across the strategies of the players.

*Saïd Business School, University of Oxford, Park End Street, Oxford OX1 1HP, United Kingdom; email: Dolores.Romero-Morales@sbs.ox.ac.uk

${ }^{\dagger}$ Department of Quantitative Economics, Maastricht University, P.O. Box 616, 6200 MD Maastricht, The Netherlands; email: D.Vermeulen@KE.unimaas.nl 
As a first leading (simple) example, one may think of the following congestion game. Suppose there are 10 tourists visiting city $C$. City $C$ has two hotels, hotel $X$ and hotel $Y$. Both hotels have room for 7 guests, so both hotels together can accommodate all tourists. The preference of each tourist is to stay in a hotel with as few other tourists as possible. Clearly, in each equilibrium 5 tourists stay in hotel $X$ and 5 tourists stay in hotel $Y$.

However, rooms have to be booked in advance. And both hotels have several ways to make a reservation, such as by phone, by fax, or via internet. Each tourist has two strategies (namely make a reservation in hotel $X$, or make a reservation in hotel $Y$ ), but the feasibility of executing such a strategy does not depend on this strategy alone, but also on the actions taken by the other tourists. If everyone else books in hotel $Y$, booking in hotel $X$ is a very good strategy. However, as soon as 7 other tourists decide to book in hotel $X$, doing this as well results in an infeasible allocation. And a tourist cannot tell the difference in advance.

In this paper we consider an infeasible allocation as an undesirable outcome for all parties concerned. There are many ways to explicitly model undesirability of infeasible allocation. We can for example add extra stages to the basic game. In the hotel example one could build in an extra renegotiation stage in which the hotels communicate with each other and redirect tourists. This implies that extra time and effort is wasted (the hotel management has to make extra phone calls and the tourists have to carry their suitcases over to the other hotel), which will be avoided in equilibrium. Another way to redefine the basic game is to incorporate the extra costs directly in the utility function of the tourists.

However, no matter how we model infeasibilities, the aim is always to augment the game in such a way that the, let us say, natural equilibria indeed become equilibria, and that infeasible allocations are not chosen in equilibrium. In the congestion game, whether we add extra stages to the game or penalize infeasibilities, the aim is to augment the game in such a way that each hotel accommodates 5 tourists in each equilibrium of the augmented game. We could achieve that by, for example, saying that for a feasible allocation the utility of a tourist equals 7 minus the number of tourists that book the same hotel, while the utility of having booked an overbooked hotel equals -1 .

Provided that we augment the game appropriately, we do not need to specify how infeasibility can, might, or ought to be resolved. On the contrary our main point here is that, even though there may be many ways to resolve infeasibilities (adding more stages to the game, or use monetary punishments for infeasible choices), in equilibrium players avoid infeasibilities. It is in that sense that the specific way in which infeasibilities are treated is not of interest to us. When the players manage to orchestrate their actions and play an equilibrium, infeasibility simply does not happen as we can clearly see in the congestion 
game.

Also on a more theoretical level feasibility restrictions across player's strategies have been studied before. For example, Caron and Laye (2003) consider a Cournot oligopoly model where market demand puts constraints on the total quantity produced by all firms. In the context of congestion games, Correa, Schulz, and Stier-Moses (2004) study efficiency of Wardrop equilibria in capacitated networks, where the capacity constraints impose feasibility restrictions across flows between origin-destination pairs in the network. In the context of strategic voting, Saporiti and Tohmé (2006) consider a voting model where the assumption of single crossing imposes restrictions across individual preferences.

Our initial motivation to study constrained competitive environments comes from decentralized decision making in supply chain planning, see e.g. Cachon and Netessine (2004) for non-cooperative games in supply chain analysis. We consider a two-level supply chain composed by suppliers at the first level and buyers at the second level. The buyers are faced with deterministic and dynamic external demand over a planning horizon, and to satisfy this demand they request inputs from the suppliers. Both suppliers and buyers face linear production and inventory holding costs as well as production capacities. This supply chain functions in a decentralized manner where information such as unit costs and capacities are only locally known. It is evident that when the suppliers plan their production, they will, in general, be constrained by the plan of the buyers. Indeed, the demand suppliers need to satisfy, and its timing, depends on the amount they get granted from the buyers' requests. Similarly, the plan of the buyers is constrained by the production decisions taken by the suppliers.

Cachon and Netessine argue that the game theoretic analysis of such models is problematic. In this paper we show that nevertheless equilibria in competitive environments under constraints across players' strategies do exist. However, Kakutani's Fixed Point theorem cannot be applied directly in this context. In particular, best replies against a given strategy profile may not be feasible. We devise a new fixed point correspondence to deal with the feasibility issue. Under the usual conditions, upper semicontinuity and convex-valuedness of best response correspondences, we show the existence of Nash equilibrium.

In the context of decentralized decision making in a supply chain, the upstream planning is one of the most well-known coordination schemes, see Bhatnagar, Chandra, and Goyal (1993). In the one-supplier one-buyer setting, the buyer plans his production and passes his plan upstream the supply chain, i.e., to the supplier. The supplier has to plan his production according to the requirements defined by the plan of the buyer. Dudek and Stadtler (2005) aim to improve this mechanism and propose a coordination scheme based 
on negotiations having as a starting point the upstream solution. In our competitive supply chain setting, where there will be several suppliers for the same product, a distribution center manages the inventory of the inputs and the corresponding costs are shared.

The distribution center receives requests from the buyers and the only information offered by the suppliers are upper bounds on production levels in each period, and its goal is to coordinate the supply chain by means of a upstream planning. However, in the presence of multiple suppliers, the upstream mechanism is a whole class depending on how the requirements of the buyers are allocated to the suppliers. We propose a plausible allocation based on fulfilling the requirements of the buyers as late as possible, thus minimizing the (shared) inventory costs. We show that, for this upstream planning mechanism, a Nash equilibrium exists. However, we illustrate with an example that the centralized solution is not, in general, an equilibrium, suggesting that regulation may be needed.

This paper is organized as follows. In Section 2 we introduce a game played in a constrained environment. Under the assumption of convex-valuedness and upper semicontinuity of the best response correspondence, we show the existence of a Nash equilibrium. In Section 3, we present the decentralized supply chain setting that motivated this work. In Section 4, we propose an upstream coordination mechanism based on (shared) inventory costs minimization, and show that for this constrained environment Nash equilibrium exists. In Section 5 we illustrate with examples that the centralized planning is not, in general, a Nash equilibrium and that the equilibrium is not unique. We derive an upper bound on the cost difference between the centralized solution and any equilibrium in which buyers request inputs according to the centralized solution. Section 6 concludes and addresses issues for further research.

\section{Existence of equilibrium in constrained environments}

In theorem 1 of this section we state and prove a generalization of the theorem of Nash $(1950,1951)$. The original theorem of Nash says that every $n$-person game in normal form has at least one equilibrium. In the normal form setting each player is supposed to make a choice from a set of mixed strategies. This choice can and has to be made independently of the other players. In our generalization we explicitly allow for constraints across the strategy spaces of the players of the game. This means that feasibility of a player's choice of strategy, in contrast with the normal form setting, may depend on the choices made by other players. As we already argued, such restrictions across strategies arise naturally in many situations.

The proof of theorem 1 is based on the fixed point theorem of Kakutani (1941). In fact theorem 1 can also be shown to be a special case of the main theorem of Debreu (1952) showing the existence of a social equilibrium in generalized games, see also Arrow and 
Debreu (1954) for a particular case of this result. The proof of Debreu is based on a fixed point theorem of Eilenberg and Montgomery (see e.g. Eilenberg and Montgomery (1946) or McLennan (1989)) for correspondences with contractible values, whose proof in its turn uses advanced techniques from algebraic topology. However, since in our particular setting we developed a novel technique to derive theorem 1 relatively easily from the more widely known result of Kakutani (1941), we decided to present a line of proof that is based on Kakutani's result.

We first formally define our model. Let $N=\{1, \ldots, n\}$ be a finite set of players. Each player $i$ has a finite set $A_{i}$ of one-dimensional decision variables at his disposal. A strategy of player $i$ is a vector

$$
x_{i}=\left(x_{i a}\right)_{a \in A_{i}}
$$

in $\mathbb{R}^{A_{i}}$. However, because we want to have feasibility restrictions explicitly available in our model, not all combinations $x=\left(x_{i}\right)_{i \in N}$ of strategy choices can be realized. Thus, let

$$
\mathcal{D} \subset \prod_{i \in N} \mathbb{R}^{A_{i}}
$$

be the collection of feasible strategy combinations. We assume that $\mathcal{D}$ is nonempty, compact and convex. A nonempty, compact and convex set $\mathcal{D}$ of feasible strategy combinations is called a constrained environment.

Best Responses Consider player $j$. Given a strategy combination $x=\left(x_{i}\right)_{i \in N}$ the set of feasible responses of player $j$ is

$$
\mathcal{D}_{j}(x):=\left\{y \in \mathcal{D} \mid y_{i}=x_{i} \text { for all } i \neq j\right\}
$$

Suppose a best response correspondence $B R_{j}: \mathcal{D} \rightarrow \mathcal{D}$ is given for player $j$ such that

(i) the graph of $B R_{j}$ is a closed subset of $\mathcal{D} \times \mathcal{D}$, and

(ii) for each $x \in \mathcal{D}, B R_{j}(x)$ is a non-empty subset of $\mathcal{D}_{j}(x)$.

Notice that we slightly deviate from the usual terminology. In the classical normal form setting best responses are a subset of the space of strategy profiles. Because of the restrictions on the strategy space that we add to the original model this is no longer the case in our setting. Therefore the usual tools like the fixed point Theorem of Kakutani no longer apply.

Definition 1. The object $G=\left\langle\mathcal{D},\left(B R_{i}\right)_{i \in N}\right\rangle$ is called a game played in a constrained environment.

Player $i$ is said to behave optimally when $x$ is an element of $B R_{i}(x)$. This justifies the following definition. 
Definition 2. A strategy profile $x \in \mathcal{D}$ is called a Nash equilibrium of the game in a constrained environment $G=\left\langle\mathcal{D},\left(B R_{i}\right)_{i \in N}\right\rangle$ if for all players $i$ we have that $x$ is an element of $B R_{i}(x)$.

A game played in a constrained environment is a rather abstract notion, and a few remarks are in order. When the set $\mathcal{D}$ of feasible strategy profiles is a Cartesian product of sets $F_{i} \subset \mathbb{R}^{A_{i}}$, where $F_{i}$ is the set of feasible strategies of player $i$, we have a conventional non-cooperative game in strategic form and it is clear how the game is played. First each player chooses a strategy $x_{i} \in F_{i}$ and an outcome $x=\left(x_{i}\right)_{i \in N}$ is realized. Payoff functions or best response sets specify how each player evaluates this outcome.

Now, a game played in a constrained environment is played in the same way (each player $i$ chooses a strategy $x_{i} \in \mathbb{R}^{A_{i}}$ ) but there is an extra complication: possibly the resulting outcome $x$ is not feasible. As said before, it is not explicitly modeled in our setting how the conundrum of infeasibility should be resolved. However, no matter how infeasibility is resolved, in equilibrium infeasibility does not occur (at least, when infeasibilities are treated correctly) and we know what the equilibria should be, irrespective of the way we resolve infeasible choices. For instance, in the congestion game described in the introduction the equilibria are those strategy profiles in which each hotel gets 5 reservations. Still, just like in this game, infeasible choices are possible. Hotels can be overbooked, flights may get canceled, and in general demand may exceed supply in many situations. And the feasibility of you making a reservation in hotel $X$ depends on the choices of others: overbooking is a feature of the entire profile of reservations by all individuals, it is not a feature of your choice alone. This phenomenon is what we try to model here.

Another point we wish to stress is that the strategy sets $A_{i}$ may well come from an extensive form game, so that the above model does not restrict us to simultaneous-move one-shot games. See Brandenburger and Stuart (2007) for a similar argument and for example Kuhn (1953) or Kohlberg and Mertens (1986) for more detailed discussions of the strategic equivalence of strategic form games and extensive form games.

We now turn to the existence of Nash equilibrium for games played in a constrained environment. Let $\mathcal{D}^{n}$ be the $n$-fold product of the set $\mathcal{D}$. An element $\left(x^{i}\right)_{i \in N}$ of $\mathcal{D}^{n}$ is called symmetric if $x^{i}=x^{j}$ for all players $i$ and $j$. Furthermore, let $\sigma$ be the cyclic permutation of the player set $N$ defined by

$$
\sigma(i):= \begin{cases}i+1 & \text { if } i \neq n \\ 1 & \text { if } i=n .\end{cases}
$$

Define the map $\phi: \mathcal{D}^{n} \rightarrow \mathcal{D}^{n}$ by

$$
\phi\left(\left(x^{i}\right)_{i \in N}\right):=\prod_{i=1}^{n} B R_{\sigma^{-1}(i)}\left(x^{\sigma^{-1}(i)}\right) .
$$


Lemma 1. Any fixed point of $\phi$ is symmetric.

Proof. Suppose that $\left(x^{i}\right)_{i \in N}$ is a fixed point of $\phi$. Because $\sigma$ is cyclic, it is sufficient to show that $x_{j}^{i}=x_{j}^{\sigma(i)}$ for all $i$ and $j$. Since $\left(x^{i}\right)_{i \in N}$ is a fixed point of $\phi$, we know from the definition of $\phi$ that

$$
x^{\sigma(i)} \in B R_{i}\left(x^{i}\right) \text { for all } i \text {. }
$$

Therefore, since $B R_{i}\left(x^{i}\right)$ is a subset of $\mathcal{D}_{i}\left(x^{i}\right)$, we know that $x_{j}^{\sigma(i)}=x_{j}^{i}$ whenever $j \neq i$. Thus we can derive the desired equality for all $i$ and $j$ with $j \neq i$. However, for $j=i$ we get from the equalities we already deduced that

$$
x_{i}^{\sigma(i)}=x_{i}^{\sigma^{2}(i)}=\cdots=x_{i}^{\sigma^{n}(i)}=x_{i}^{i} .
$$

Using lemma 1 we can prove existence of Nash equilibrium for games that are played in constrained environments, if the best responses are convex.

Theorem 1. Let $G$ be a game played in a constrained environment. If for each $x \in \mathcal{D}$ and $j \in N, B R_{j}(x)$ is convex, then the game $G$ has at least one Nash equilibrium.

Proof. Apply the Fixed Point theorem of Kakutani to $\phi$. This yields a point $\left(x^{i}\right)_{i \in N}$ with $x^{\sigma(i)} \in B R_{i}\left(x^{i}\right)$ for all $i$. However, by lemma 1 , we know that there is a strategy combination $x \in \mathcal{D}$ such that $x^{i}=x$ for all $i$. Hence, $x \in B R_{i}(x)$ for all $i$, and $x$ is a Nash equilibrium of the game $G$.

Computation of all Nash equilibria, or even a single Nash equilibrium, may be a complicated task. Even though a single player, given the strategies of his opponents, faces a simple one-person decision problem, Nash equilibrium requires that players play optimally in an orchestrated fashion. Even for the simplest case, finite two player strategic form games (bimatrix games) it is still an open question whether computation of Nash equilibrium can be achieved in polynomial time. Savani and von Stengel (2006) recently showed that the most notable algorithm for computation of a single Nash equilibrium, the Lemke-Howson algorithm, see Lemke and Howson (1964), has a worst-case exponential running time. Computing a Nash equilibrium for more than two players involves solving higher order polynomial (in)equalities, and is a hard problem. Recently, Daskalakis, Goldberg and Papadimitriou (2006) have shown that computation of a Nash equilibrium with 4 or more players is PPAD complete ${ }^{1}$. Chen and Deng (2005) have shown the PPAD completeness of finding a Nash equilibrium for bimatrix games.

\section{The supply chain planning framework}

In this section we present the application that motivated this work. We consider a twolevel supply chain composed by suppliers at the first level and buyers at the second level,

\footnotetext{
${ }^{1}$ The class PPAD is introduced in Papadimitriou (1994). PPAD stands for Polynomial Parity Argument (Directed case). It is a class of search problems similar to, but not equal to, the class NP.
} 
both keeping most of their parameters, such as unit costs and capacities, locally, see e.g. Dudek and Stadtler (2005) for the one-supplier one-buyer setting. The buyers are faced with deterministic and dynamic external demand over a planning horizon, and to satisfy this demand they source inputs from the suppliers. Since we are dealing with a competitive environment where there are multiple suppliers for the same product, we coordinate this supply chain by means of a distribution center that is in charge of making a production plan while taking into account the information provided by each of the members. In the following we describe in detail the parameters associated to this model.

Let $S$ be the set of suppliers and $B$ be the set of buyers that have to satisfy a demand pattern over a discrete planning horizon of $T$ periods. At each period $t=1, \ldots, T$ each buyer $b \in B$ faces a demand of $d(b, t) \geq 0$ units for the so-called end product.

In order to produce one unit of the end product each buyer $b \in B$ needs one unit of a so-called input product, which he can subsequently transform into the end product at a cost of $c(b, t)$ when production takes place at period $t$.

The buyers should satisfy the demand exactly in time. Therefore, when needed buyers will keep the end product in stock, where the unit inventory holding costs for buyer $b$ at period $t$ are equal to $h(b, t)$.

Upstream in the supply chain each supplier $s \in S$ can produce the input product. The unit production costs faced by supplier $s$ at period $t$ are equal to $c(s, t)$. The capacity of supplier $s$ at period $t$ is equal to $m(s, t)$.

Finally, the input product can only be held in stock at the distribution center, where the unit inventory holding at period $t$ are equal to $g(t)$. These costs are shared equally by the buyers and the suppliers.

In order to acquire the input product buyer $b$ in $B$ places an order $r(b, t) \geq 0$ at the distribution center for each period $t$. Following the upstream planning, see Bhatnagar, Chandra, and Goyal (1993), the distribution center promises to deliver these requirements just in time. Independently and simultaneously, supplier $s$ in $S$ reports quantities $q(s, t)$ to the distribution center representing the maximal amount of input product he is willing to produce at period $t$. The distribution center has two tasks: checking for the feasibility of the ask/bid profile, and allocating the requests of the buyers to the suppliers. Recall that the first task is necessary since we are dealing with constraints across the players' strategies. Once these two tasks have been performed the players can calculate their payoffs. In the following, we discuss the feasibility and the form of the payoffs for any feasible upstream mechanism. In section 4 we discuss in detail an upstream mechanism based on minimizing the shared inventory holding costs at the distribution center. 
Note that buyers and suppliers make a plan at the start of the planning horizon for the entire planning horizon, all orders and reports are submitted before the beginning of the planning horizon. In the same fashion, the distribution center returns the plan to the suppliers at the start of the planning horizon. This is a one-shot game, in contrast to dynamic versions, where a game is played in period $t$, and the output of this game is known before playing game in period $t+1$.

\section{Feasibility of ask/bid profiles}

Given the specification of each requirement $r(b, t)$ by the buyers and each capacity $q(s, t)$ by the suppliers, the distribution center first checks feasibility of the ask/bid profile as follows. Write

$$
D(b, t):=d(b, 1)+\cdots+d(b, t)
$$

the total demand for buyer $b$ up to and including period $t$. Similarly we write

$$
R(b, t):=r(b, 1)+\cdots+r(b, t),
$$

the total requirement of buyer $b$ up to and including period $t$, and

$$
Q(s, t):=q(s, 1)+\cdots+q(s, t)
$$

the total reported capacity of supplier $s$ up to and including period $t$. The feasibility restrictions we impose can now be written as follows.

For each buyer $b \in B$, for each supplier $s \in S$, and for all $t=1, \ldots, T$

$$
R(b, t) \geq D(b, t) \quad \text { and } \quad m(s, t) \geq q(s, t) .
$$

These conditions reflect demand satisfaction for the end product and the production restrictions on the supply side, respectively. Moreover we require that

$$
\sum_{s \in S} Q(s, t) \geq \sum_{b \in B} R(b, t)
$$

for all $t=1, \ldots, T$. These conditions ensure demand satisfaction for the input product. The set of feasible ask/bid profiles $(q, r)$ is denoted by $D$. To show the main result of this paper, we assume that $D$ is not empty, i.e., there exists at least one feasible ask/bid profile. In particular, we will assume throughout the rest of the paper that the ask/bid profile $(m, d)$, in which each buyer reports his demand and each supplier reports his maximum capacity, is feasible. This is a very mild condition and it does not preclude the infeasibility of other ask/bid profiles. As already said in the introduction, our proof of existence of an equilibrium will be the same, independently of the way these infeasibilities are resolved, provided that we augment the game appropriately. One choice that may 
be very appealing here is the use of a penalization function every time an ask/bid profile falls outside the feasible region $D$, where the penalization is big enough to ensure that infeasible profiles are never chosen in equilibrium.

\section{Feasibility of coordination mechanisms}

For each feasible ask/bid profile $(q, r)$ in $\mathcal{D}$ the distribution center announces a binding coordination arrangement. The way this binding coordination arrangement is constructed is public knowledge to all the players in the supply chain. As said before, each buyer $b$ has to pick up $r(b, t)$ units from the distribution center during period $t$ and to transform this amount into end product. For supplier $s$ and period $t$, the distribution center needs to specify

$$
x_{s}(q, r, t)
$$

such that supplier $s$ is now bound to produce $x_{s}(q, r, t)$ units in period $t$. Thus, a coordination arrangement, or mechanism, is a collection

$$
x=\left(x_{s}\right)_{s \in S}
$$

of continuous functions $x_{s}: \mathcal{D} \times T \rightarrow \mathbb{R}$, the delivery schedules for the suppliers to the distribution center. A mechanism must satisfy the following conditions. Write

$$
\begin{aligned}
X_{s}(q, r, t) & =x_{s}(q, r, 1)+\cdots+x_{s}(q, r, t) \\
X(q, r, t) & =\sum_{s \in S} X_{s}(q, r, t) \\
x(q, r, t) & =\sum_{s \in S} x_{s}(q, r, t) .
\end{aligned}
$$

We require the following set of conditions. For each supplier $s \in S$,

$$
x_{s}(q, r, t) \leq q(s, t)
$$

for all $t=1, \ldots, T$. Further, the total stock in the distribution center should always be non-negative. So, for all $t=1, \ldots, T$.

$$
X(q, r, t) \geq \sum_{b \in B} R(b, t)
$$

\section{Total production and inventory costs}

As said before inventory costs at the distribution center are supposed to be shared equally by all buyers and suppliers alike. So, if we write

$$
G(q, r, t):=X(q, r, t)-\sum_{b \in B} R(b, t)
$$


for the total stock available at the distribution center at period $t$, the costs associated with the binding coordination arrangement $\left(x_{s}(q, r, t)\right)_{t=1}^{T}$ for supplier $s$ are

$$
V_{s}(q, r):=\sum_{t=1}^{T} c(s, t) x_{s}(q, r, t)+\frac{1}{|B|+|S|} \sum_{t=1}^{T} g(t) G(q, r, t)
$$

The first term is equal to the total production costs given the binding coordination arrangement imposed by the distribution center. The second term is the part of the inventory costs associated with the input product that supplier $s$ is supposed to pay.

Similarly, for buyer $b$, his costs are equal to

$$
V_{b}(q, r):=\sum_{t=1}^{T} c(b, t) r(b, t)+\frac{1}{|B|+|S|} \sum_{t=1}^{T} g(t) G(q, r, t)+\sum_{t=1}^{T} h(b, t)(R(b, t)-D(b, t)) .
$$

We may observe that, as in a standard non-cooperative game, a player would need to model the actions of the rest of the players to be able to calculate its own payoffs. Once these actions are defined, this player only needs two ingredients to calculate its payoff, namely its own cost structure and the agreement returned by the distribution center, which is public information to every player.

So far we have only addressed the decisions of the distribution center given an ask/bid profile $(q, r)$. However, when choosing a strategy, buyers and suppliers are thus confronted with an entire range of opportunities by changing their stated requirements or capacities. The set of feasible responses of supplier $s$ given the ask/bid profile $(q, r)$ is the set

$$
\mathcal{D}_{s}(q, r):=\left\{\left(q^{\prime}, r\right) \in \mathcal{D} \mid q_{s^{\prime}}^{\prime}=q_{s^{\prime}} \text { for all } s^{\prime} \neq s\right\}
$$

The set of best responses of supplier $s$ given the ask/bid profile $(q, r)$ is the set

$$
B R_{s}(q, r):=\left\{\left(q^{\prime}, r\right) \in \mathcal{D}_{s}(q, r) \mid V_{s}\left(q^{\prime}, r\right) \leq V_{s}\left(q^{\prime \prime}, r\right) \text { for all }\left(q^{\prime \prime}, r\right) \in \mathcal{D}_{s}(q, r)\right\}
$$

In the same way we can define the sets $\mathcal{D}_{b}(q, r)$ and $B R_{b}(q, r)$ for each buyer $b$ in $B$.

The next lemma shows that for any upstream mechanism $G=\left\langle\mathcal{D},\left(B R_{b}\right)_{b \in B},\left(B R_{s}\right)_{s \in S}\right\rangle$ is a game played in a constrained environment. At least $\mathcal{D}$ is non-empty by assumption, and clearly compact and convex, because suppliers have a maximum capacity and all restrictions are linear.

Lemma 2. $G=\left\langle\mathcal{D},\left(B R_{b}\right)_{b \in B},\left(B R_{s}\right)_{s \in S}\right\rangle$ is a game played in a constrained environment.

Proof. We need to show that the graphs of the correspondences $B R_{s}$ and $B R_{b}$ are closed. Moreover, both correspondences are non-empty valued. 
We will show that the graph of the correspondence $B R_{s}$ is closed. A similar proof will hold for $B R_{b}$. Suppose that for every $k$ we have a

$$
\left(q^{\prime k}, r^{k}\right) \in B R_{s}\left(q^{k}, r^{k}\right)
$$

and that $\left(\left(q^{\prime k}, r^{k}\right),\left(q^{k}, r^{k}\right)\right) \rightarrow\left(\left(q^{\prime}, r\right),(q, r)\right)$ as $k \rightarrow \infty$. We will show that $\left(q^{\prime}, r\right)$ is an element of $B R_{s}(q, r)$. Take a point $\left(q^{\prime \prime}, r\right)$ in $\mathcal{D}_{s}(q, r)$. Notice that the set $\mathcal{D}$ is polyhedral. Thus we know, see e.g. Cook et al. (1986), that there exist points $\left(q^{\prime \prime k}, r^{k}\right)$ in $\mathcal{D}_{s}\left(q^{k}, r^{k}\right)$ that converge to $\left(q^{\prime \prime}, r\right)$ as $k \rightarrow \infty$. However, since $\left(q^{\prime k}, r^{k}\right)$ is an element of $B R_{s}\left(q^{k}, r^{k}\right)$, we know for all $k$ that

$$
V_{s}\left(q^{\prime k}, r^{k}\right) \leq V_{s}\left(q^{\prime \prime k}, r^{k}\right) .
$$

Hence, $V_{s}\left(q^{\prime}, r\right) \leq V_{s}\left(q^{\prime \prime}, r\right)$ by continuity, and the desired result holds. Non-emptiness of the values of both $B R_{b}$ and $B R_{s}$ follows from the observation that these values are given by the minimization of a continuous function over a compact set.

The goal now is to define the way the production planning is constructed by the distribution center, such that the best response correspondences are convex. We propose a very appealing alternative, based on minimizing the shared inventory holding costs.

\section{Existence of equilibria for inventory cost minimization planning}

In the following we will discuss a variant of the upstream planning mechanism for which we can use theorem 1 to show the existence of a Nash equilibrium. To define the coordination mechanism completely, it remains to describe the way the distribution center constructs a plan for the suppliers. The objective is to keep the inventory costs of the input product as low as possible. In our case this is equivalent to satisfying the requests of the buyers as late as possible. Hereafter we assume that the suppliers are put in a queue. This queue is modeled by the sequence $s(1), s(2), \ldots, s(n)$, where $s(1)$ is the supplier in the front of the queue, $s(2)$ is the second supplier in the queue, et cetera. The specific order is not crucial in what follows. The queue is mainly used as a tie breaking tool so that we can make unambiguous assignments of quantities to players within a period. Thus we assume without loss of generality that $s(i)=i$ for all $i$.

Given $\xi, \rho \in \mathbb{R}^{T}$, let $\theta(\xi, \rho)$ be the optimal value of the linear programming problem

$$
\operatorname{minimize} \sum_{t=1}^{T} g(t) I_{t}
$$

subject to

$$
\begin{array}{rlrl}
X_{t}-I_{t}+I_{t-1} & =\rho_{t} & t=1, \ldots, T \\
X_{t} & \leq \xi_{t} & t=1, \ldots, T \\
I_{0} & =0 & \\
X_{t}, I_{t} & \geq 0 & t=1, \ldots, T
\end{array}
$$


where $X_{t}$ is the production level in period $t$ and $I_{t}$ is the inventory level at the end of period $t$. The goal of this problem is to satisfy the request pattern $\rho$ subject to the production capacities $\xi_{t}$, such that the total inventory holding costs $\sum_{t=1}^{T} g(t) I_{t}$ are minimized. Since the production variables $X_{t}$ are not in the objective function, it is easy to show that we produce the requirements $\rho_{t}$ as late as possible, given the capacities $\xi_{t}$.

Once the distribution center has collected the strategies chosen by the players and has checked that the $(q, r)$ profile is feasible, it defines $\xi_{t}=\sum_{s \in S} q(s, t)$ and $\rho_{t}=\sum_{b \in B} r(b, t)$, and computes $\theta(\xi, \rho)$ obtaining the minimal inventory holding costs to satisfy the requests of the buyers given the capacities offered by the suppliers. The optimization problem also provides the aggregate production vector $X \in \mathbb{R}^{T}$. To finalize the planning, the distribution center needs to disaggregate the amounts $X_{t}$ to obtain $x_{s}(q, r, t)$. Using the order defined in the set of suppliers, this disaggregation is well-defined and unique. It starts by letting the first supplier produce to full capacity if needed, otherwise, it will produce only what is needed. If this supplier has produced to full capacity then we make a similar construction with supplier 2 , and we continue until the aggregate production has been assigned to the suppliers. In Appendix A, we derive a formal recursive expression for $x_{s}(q, r, t)$.

We now prove the existence of Nash equilibria in the inventory cost minimization planning mechanism by means of theorem 1 . In the following we first show that the best reply correspondences $B R_{b}$ are convex.

Lemma 3. The correspondence $B R_{b}$ has convex values.

Proof. Take a buyer $b$ in $B$. His best response set $B R_{b}(q, r)$ given a feasible ask/bid profile $(q, r)$ is given as the set of reports $(r(b, t))_{t=1}^{T}$ that minimize the objective function $V_{b}(q, r):=\sum_{t=1}^{T} c(b, t) r(b, t)+\frac{1}{|B|+|S|} \sum_{t=1}^{T} g(t) G(q, r, t)+\sum_{t=1}^{T} h(b, t)(R(b, t)-D(b, t))$.

over the set

$$
\mathcal{D}_{b}(q, r):=\left\{\left(q, r^{\prime}\right) \in \mathcal{D} \mid r_{b^{\prime}}^{\prime}=r_{b^{\prime}} \text { for all } b^{\prime} \neq b\right\} .
$$

We may observe that the first and the last terms of the objective functions are linear in $r(b, t)$. Moreover, since the distribution center minimizes the total shared inventory costs, we know that the second term is equal to $\frac{1}{|B|+|S|} \sum_{t=1}^{T} g(t) G(q, r, t)=\frac{1}{|B|+|S|} \theta(\xi, \rho)$ where $\xi_{t}=\sum_{s \in S} q(s, t)$ and $\rho_{t}=\sum_{b \in B} r(b, t)$. Therefore, it remains to show that the function $\theta(\xi, \rho)$ is a convex function in $\rho$. This easily follows after writing the dual formulation of the linear programming model defining $\theta(\xi, \rho)$. We have that

$\theta(\xi, \rho)=\max \left\{\sum_{t=1}^{T}\left(\lambda_{t} \rho_{t}-\mu_{t} \xi_{t}\right): \lambda_{t}, \mu_{t} \geq 0, \lambda_{t}-\mu_{t} \leq 0,-\lambda_{t}+\lambda_{t+1} \leq g(t),-\lambda_{T} \leq g(T)\right\}$. 
Therefore, $\theta(\xi, \rho)$ as a function of $\rho$ can be seen as the maximization of linear functions on a polytope, which is a convex function.

Unfortunately, for the suppliers we cannot prove a similar result.

Example 1. Consider a single supplier and a single buyer. The supplier has three days to produce one unit of input product for the buyer. Each day his capacity is equal to 2. A reported capacity $\left(q_{1}, q_{2}, q_{3}\right)$ for the supplier is feasible if each $q_{i}$ is between 0 and 2 , and moreover $q_{1}+q_{2}+q_{3} \geq 1$. Graphically this can be represented by the cube $[0,2]^{3}$ with one corner chopped off.

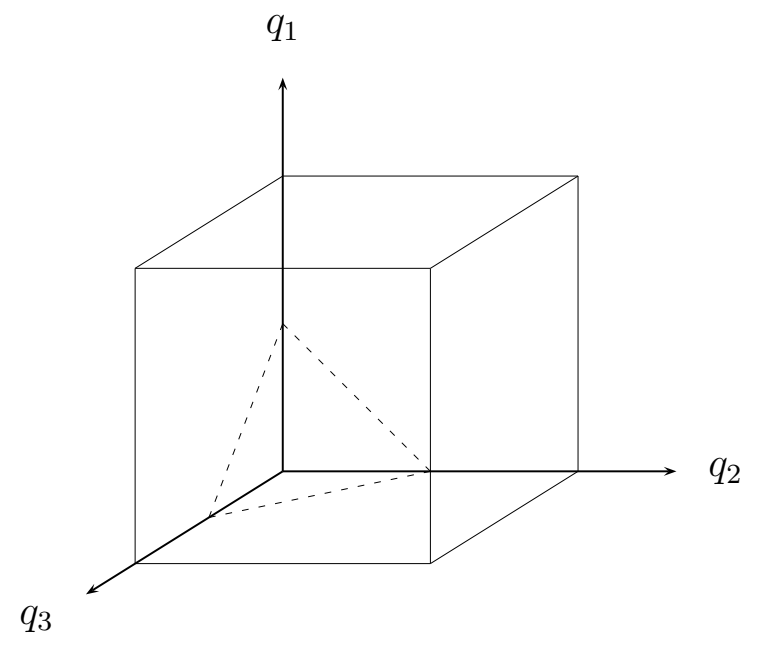

Now, given a feasible reported capacity $\left(q_{1}, q_{2}, q_{3}\right)$, our upstream planning mechanism returns the schedule

$$
\left(x_{1}, x_{2}, x_{3}\right)= \begin{cases}(0,0,1) & \text { if } q_{3} \geq 1 \\ \left(0,1-q_{3}, q_{3}\right) & \text { if } q_{3}<1 \text { and } q_{2}+q_{3} \geq 1 \\ \left(1-q_{2}-q_{3}, q_{2}, q_{3}\right) & \text { else. }\end{cases}
$$

Moreover, suppose that the production and inventory costs are such that producing in periods 1 and 3 is equally expensive, while production in period 2 is very costly. We then have $c(s, 1)+\frac{1}{2} \times(g(1)+g(2))=c(s, 3)<c(s, 2)+\frac{1}{2} \times g(2)$. Thus, the set of best replies is $\mathcal{S} \cup \mathcal{T}$ with

$$
\mathcal{S}=\left\{\left(q_{1}, 0, q_{3}\right) \mid q_{1}+q_{3} \geq 1,0 \leq q_{3} \leq 1,0 \leq q_{1} \leq 2\right\}
$$

and

$$
\mathcal{T}=\left\{\left(q_{1}, q_{2}, q_{3}\right) \mid 1 \leq q_{3} \leq 2,0 \leq q_{1} \leq 2,0 \leq q_{2} \leq 2\right\}
$$

where we avoid producing in period 2 since it is more expensive than producing in any of the other periods. Graphically this is the shaded area $\mathcal{S}$ together with the box $\mathcal{T}$ depicted in the front of the picture below. 


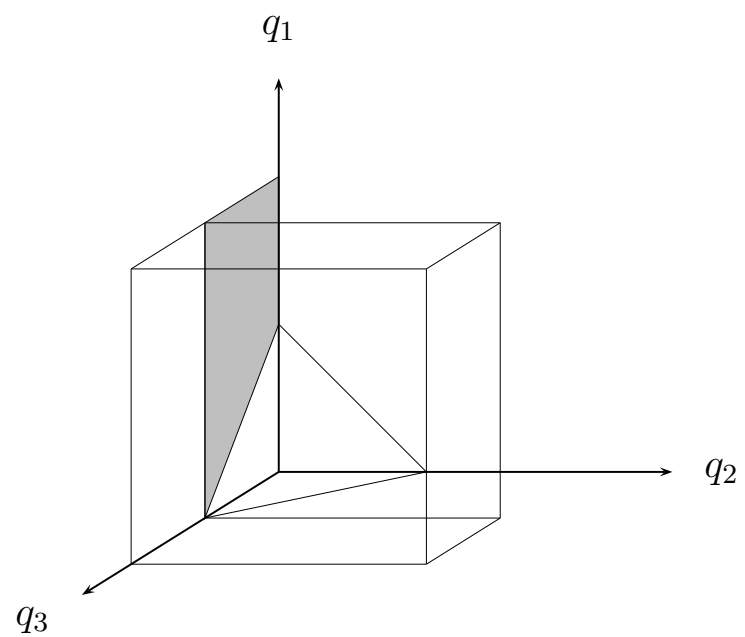

However, this is clearly not a convex set.

In the following we construct a subcorrespondence of $B R_{s}(q, r)$. First, observe that given a feasible ask/bid profile in the above example, say $(q, r) \in \mathcal{D}$, supplier $s$ gets the same plan back from the distribution center when reporting $q^{\prime}$ or $x_{s}\left(q^{\prime}, r, \cdot\right)$. Therefore, if $\left(q^{\prime}, r\right) \in B R_{s}(q, r)$, then $\left(x_{s}\left(q^{\prime}, r, \cdot\right), r\right) \in B R_{s}(q, r)$. In the above example it can easily be seen that convexity is not a problem when we only consider best responses of the latter type. This observation is the motivation for the following definitions.

Definition 3. Let $(q, r)$ be an element of $\mathcal{D}$ and let $s$ be a supplier in $S$. An element $\left(q^{\prime}, r\right)$ of $B R_{s}(q, r)$ is called a minimal best response of supplier $s$ if supplier $s$ has reported such that $x_{s}\left(q^{\prime}, r, t\right)=q^{\prime}(s, t)$, for all $t$. The set of minimal best responses of supplier $s$ given $(q, r)$ is denoted by $M_{B}(q, r)$.

$\triangleleft$

In the following we show the existence of Nash equilibria in our supply chain application. To prove this result we need lemma B.3 in Appendix B, showing that the minimal best responses correspondences $M B R_{s}$ are convex.

Theorem 2. The game $\left\langle\mathcal{D},\left(B R_{b}\right)_{b \in B},\left(M B R_{s}\right)_{s \in S}\right\rangle$ has a Nash equilibrium, and therefore the game $\left\langle\mathcal{D},\left(B R_{b}\right)_{b \in B},\left(B R_{s}\right)_{s \in S}\right\rangle$ also has Nash equilibrium.

Proof. By theorem 1 and lemmas 3 and B.3, it suffices to show that the game $\left\langle\mathcal{D},\left(B R_{b}\right)_{b \in B},\left(M B R_{s}\right)_{s \in S}\right\rangle$ is played in a constrained environment. By lemma 2 we already know that the graph of $B R_{b}$ is closed and that its values are nonempty. In the following we study similar properties for $M B R_{s}$.

First we will show that the graph of $M B R_{s}$ is closed. To see this, notice that, by the definition of a minimal best response, the graph of $M B R_{s}$ is equal to the intersection of the graphs of $B R_{s}$ and the correspondence $\varphi_{s}$ defined by

$$
\varphi_{s}(q, r)=\left\{\left(q^{\prime}, r\right) \in \mathcal{D}_{s}(q, r) \mid x_{s}\left(q^{\prime}, r, t\right)=q^{\prime}(s, t), \text { for all } t\right\}
$$


The graph of $B R_{s}$ is closed according to lemma 2, while the graph of $\varphi_{s}$ is closed by the maximum theorem (see e.g. Berge (1966)), because $\mathcal{D}_{s}$ is a continuous correspondence and $x_{s}$ is a continuous function. Hence, the graph of $M B R_{s}$ is also closed.

Next, take an ask/bid profile $(q, r)$ in $\mathcal{D}$. By lemma 2 we know that $B R_{s}(q, r)$ is not empty, so we can take a $\left(q^{\prime}, r\right) \in B R_{s}(q, r)$. Define $q^{\prime \prime}$ by, for $t \in T, q^{\prime \prime}(\ell, t)=q(\ell, t)$ for all $\ell \neq s$, and $q^{\prime \prime}(s, t)=x_{s}\left(q^{\prime}, r, t\right)$. We will show that $\left(q^{\prime \prime}, r\right)$ is an element of $M B R_{s}(q, r)$.

It is straightforward to check that $\left(q^{\prime \prime}, r\right)$ is an element of $\mathcal{D}_{s}(q, r)$. Furthermore, since $x_{\ell}\left(q^{\prime}, r, t\right) \leq q^{\prime \prime}(\ell, t) \leq q^{\prime}(\ell, t)$ clearly holds for all $\ell \in S$, it is easy to see that $x_{\ell}\left(q^{\prime}, r, t\right)=$ $x_{\ell}\left(q^{\prime \prime}, r, t\right)$ for all $\ell \in S$. Thus we get that $V_{s}\left(q^{\prime}, r\right)=V_{s}\left(q^{\prime \prime}, r\right)$ and $\left(q^{\prime \prime}, r\right)$ is an element of $B R_{s}(q, r)$. Finally, $q^{\prime \prime}(s, t)=x_{s}\left(q^{\prime}, r, t\right)=x_{s}\left(q^{\prime \prime}, r, t\right)$.

Note that we do not require suppliers to report exclusively minimal best responses. We only showed that a Nash equilibrium exists in which each strategy is a minimal best response against the strategies of the opponents. However, there may well be equilibria in which sellers use strategies that are non-minimal best responses.

\section{Properties of the supply chain equilibria}

In this section we discuss some properties of the supply chain equilibria. First we show that the centralized solution - the plan that minimizes the total cost faced by both suppliers and buyers- will in general not be a Nash equilibrium. This is by the way not a feature that is restricted to upstream planning mechanisms. For virtually all mechanisms the centralized solution will often not be a Nash equilibrium. Then, we illustrate that the equilibrium is not unique in general. Finally, we derive a bound, for the buyers, on the difference between their costs in the centralized solution and any equilibrium derived from the centralized solution.

Example 2. Consider a planning horizon consisting of six time periods, i.e. $T=6$, one single supplier and one single buyer. The data are as follows. For the buyer we have

\begin{tabular}{|r|r|r|r|r|r|r|}
\hline$t=$ & 1 & 2 & 3 & 4 & 5 & 6 \\
\hline$d(b, t)=$ & 2 & 4 & 8 & 10 & 8 & 4 \\
\hline$c(b, t)=$ & 5 & 20 & 5 & 20 & 5 & 5 \\
\hline$h(b, t)=$ & 3 & 3 & 3 & 3 & 3 & 3 \\
\hline
\end{tabular}

and for the supplier we have

\begin{tabular}{|r|r|r|r|r|r|r|}
\hline$t=$ & 1 & 2 & 3 & 4 & 5 & 6 \\
\hline$c(s, t)=$ & 10 & 10 & 20 & 20 & 20 & 13 \\
\hline
\end{tabular}

The unit inventory holding costs for the input product faced by the distribution center are equal to 1 , equally paid by the supplier and the buyer $\left(\frac{1}{2}\right.$ per period each).

The centralized solution $\left(x^{*}(s, t), y^{*}(b, t)\right)$ (the feasible production plan that minimizes total cost, given that the demand is satisfied) is given by 


\begin{tabular}{|r|r|r|r|r|r|r|}
\hline$t=$ & 1 & 2 & 3 & 4 & 5 & 6 \\
\hline$x^{*}(s, t)=$ & 6 & 26 & & & & 4 \\
\hline$y^{*}(b, t)=$ & 6 & & 18 & & 8 & 4 \\
\hline$G(t)=$ & & 26 & 8 & 8 & & \\
\hline$O(t)=$ & 4 & & 10 & & & \\
\hline
\end{tabular}

where $x^{*}(s, t)$ is the amount of input product the distribution center requires the supplier to deliver at period $t, y^{*}(b, t)$ is the amount of input product the distribution center requires the buyer to collect (and subsequently transform into end product), $G(t)$ is the amount of the input product stored at the distribution center at period $t$ and $O(t)$ is the amount of end product stored at period $t$. The total cost of this production schedule equals 636, of which

$$
6 \times 10+26 \times 10+4 \times 13+\frac{1}{2}(26+8+8)=393
$$

is paid by the supplier and

$$
6 \times 5+18 \times 5+8 \times 5+4 \times 5+\frac{1}{2}(26+8+8)+3(4+10)=243
$$

is paid by the buyer.

This production schedule $\left(x^{*}(s, t), y^{*}(b, t)\right)$ can never be an equilibrium outcome of the upstream planning mechanism for the following reason. Let $(q, r)$ be a feasible ask/bid profile that has the centralized solution as an outcome of the upstream planning mechanism, i.e. $x_{s}(q, r, t)=x^{*}(s, t)$ and $r(b, t)=y^{*}(b, t)$. (Recall that in the upstream planning the distribution center simply copies the requirements of the buyer.)

However, given these requirements reported by the buyer, the supplier has a feasible bid for his capacities that results in lower costs than in the centralized solution. If the supplier reports

\begin{tabular}{|r|r|r|r|r|r|r|}
\hline$t=$ & 1 & 2 & 3 & 4 & 5 & 6 \\
\hline$q^{\prime}(s, t)=$ & 6 & 30 & & & & \\
\hline
\end{tabular}

the resulting production schedule from the upstream planning mechanism is

\begin{tabular}{|r|r|r|r|r|r|r|}
\hline$t=$ & 1 & 2 & 3 & 4 & 5 & 6 \\
\hline$x_{s}\left(q^{\prime}, r, t\right)=$ & 6 & 30 & & & & \\
\hline$G(t)=$ & & 30 & 12 & 12 & 4 & \\
\hline$O(t)=$ & 4 & & 10 & & & \\
\hline
\end{tabular}

with a total cost of 640 , of which

$$
6 \times 10+30 \times 10+\frac{1}{2}(30+12+12+4)=389
$$


is paid by the supplier and

$$
6 \times 5+18 \times 5+8 \times 5+4 \times 5+\frac{1}{2}(30+12+12+4)+3(4+10)=251
$$

is paid by the buyer. Thus, the centralized schedule can never be the outcome of a decentralized optimal choice for the supplier under our upstream planning mechanism. In this context it is interesting to note that, in the language of Brandenburger and Stuart (2007), our model will at least violate the No Externalities condition, because the maximum cost reduction the supplier can realize depends crucially on the choice of requirements the buyer submits. Thus Proposition 5.1 of Brandenburger and Stuart, which establishes the (cost) efficiency of Nash equilibrium, does not apply as we observed in this example. $\triangleleft$

In the following we will show that, for a given problem instance, there may exist more than one equilibrium, which is usually the case. We use the same data as in example 1.

Example 3. Consider the one-supplier one-buyer problem instance presented in example 1, where the buyer needs to produce exactly one unit in period 3, i.e., the vector of demands is equal to $(0,0,1)$. Suppose that the buyer can produce in any period at the same cost and that the unit inventory holding costs $h(b, t)$ are equal to the input ones, i.e., $h(b, t)=g(t)$, for all $t$. Because the input inventory holding costs are equally shared by the supplier and the buyer and the production costs at the buyer are stationary, if needed, the buyer prefers to keep the inventory at the distribution center. Therefore, given the vector of requirements $r=(0,0,1)$, it is easy to see that the following two ask/bid profiles $\left(q^{\prime}, r\right)$ and $\left(q^{\prime \prime}, r\right)$ yield an equilibrium, where $q^{\prime}=(1,0,0)$ and $q^{\prime \prime}=(0,0,1)$.

In the light of example 2, one straightforward question would be how bad Nash equilibria can be compared to the centralized planning. However, we have shown in example 3 that for a given problem instance there may be multiple equilibria. In the following proposition we focus on the buyers point of view and, in particular, on the requirements pattern obtained from the centralized solution.

Proposition 1. The difference between the costs buyer b faces in the centralized solution and the costs he pays in any equilibrium implementing the requirements pattern of buyer $b$ in the centralized solution is at most

$$
\frac{1}{|S|+|B|} \times(T-1) \times D(b, T) \times \max _{t} g(t) .
$$

Proof. Because we are using an upstream planning, the distribution center implements the requirements of the buyers. Therefore, for any buyer $b$, the difference between the centralized solution and any associated equilibrium is only in the input inventory levels, i.e., the production as well as the end-product inventory levels are the same. We will show that the two ask/bid profiles differ in at most $(T-1) \times D(b, T)$ units of inventory and the 
desired result following by noticing that the unit input inventory holding cost that buyer $b$ needs to pay in any period is at most $\frac{1}{|S|+|B|} \times \max _{t} g(t)$.

In the worst case, buyer $b$ may require everything in the last period and the suppliers may have the cheapest option for producing (including the shared unit inventory holding costs for the input product) in the first period. Therefore, the suppliers will only submit capacities in period 1 . Thus, buyer $b$ may need to pay this extra inventory holding costs, i.e., carry the total demand in inventory from period 1 until period $T$.

\section{Conclusion and further research}

In this paper we showed the existence of Nash equilibrium in game theoretic models with constraints across players' strategies. We apply the result in the context of a two-level supply chain. We showed that for the upstream mechanism where the requests are produced as late as possible, we can apply the existence result to a subcorrespondence of the best response correspondence. We also showed that in the two-level supply chain setting the centralized solution is in general not a Nash equilibrium and that the equilibrium may not be unique.

The existence result can be useful for further applications in games where the feasibility depends on the strategies of all players taken together. Such situations arise especially in contexts of decentralized decision making where local decisions can disrupt the company's planning. It may be interesting to apply our result to for example coordination of design or manufacturing divisions in a firm. Also the introduction of transfer prices in the supply chain model seems to be an interesting and non-trivial extension of our current supply chain model.

The observation that Nash equilibrium is in general not optimal seems to suggest that, when decision making is decentralized in the supply chain, that the chain may get caught in a non-optimal solution. One way to resolve this is by monetary transfers. We conjecture it is possible to improve upon the Nash equilibrium outcome by means of payment schemes or subsidies. Under exactly which circumstances is it possible to guarantee full optimality with such payment schemes? One other direction for further research would be to see whether we can apply the existence result also in a more general setting, for example allowing for setup costs in production.

\section{Acknowledgements}

The authors thank Joris van de Klundert and four anonymous referees for their insightful comments which have helped to improve the paper considerably.

\section{References}

Arrow, K.J., and G. Debreu. (1954) Existence of an equilibrium for a competitive economy, 
Econometrica $22(3), 265-290$.

Berge, C. (1966) Espaces Topologiques, Dunod, Paris (2e édition).

Bhatnagar, R., P. Chandra, and S.K. Goyal. (1993) Models for multi-plant coordination, European Journal of Operational Research 67, 141 - 160.

Brandenburger, A., and H. Stuart. (2007) Biform games, Management Science 53, 537 549.

Cachon, G.P., and S. Netessine. (2004) Game theory in supply chain analysis, In D. Simchi-Levi, S.D. Wu, and Z-J. Shen, editors, Handbook of Quantitative Supply Chain Analysis, 13 - 65, Kluwer Academic Publishers, Boston.

Caron, C., and J. Laye. (2003) A fast algorithm for capacity constrained Cournot-Nash equilibrium, Working paper.

Chen, X. and X. Deng. (2005) Settling the complexity of 2-player Nash equilibrium, Electronic Colloquium on Computational Complexity TR05-140.

Cook, W., A.M.H. Gerards, A. Schrijver, and É. Tardos. (1986) Sensitivity theorems in integer linear programming, Mathematical Programming 34, 48 - 61.

Correa, J.R., A.S. Schulz, and N.E. Stier-Moses. (2004) Selfish routing in capacitated networks, Mathematics of Operations Research 29 (4), 961 - 976.

Daskalakis, C., Goldberg, P. and C.H. Papadimitriou. (2006) The complexity of computing a Nash equilibrium, Proceedings of the $38^{\text {th }}$ Annual ACM Symposium on Theory of Computing, $71-78$.

Debreu, G. (1952) A social equilibrium existence theorem, Proceedings of the National Academy of Sciences 38, 886 - 893.

Eilenberg, S., and D. Montgomery. (1946) Fixed-point theorems for multivalued transformations, American Journal of Mathematics 68, 214 - 222.

Dudek, G., and H. Stadtler. (2005) Negotiation-based collaborative planning between supply chains partners, European Journal of Operational Research 163, 668 - 687.

Kakutani, S. (1941) A generalization of Brouwer's fixed point theorem, Duke Mathematical Journal 8, $457-459$.

Kohlberg E., and J.-F. Mertens. (1986) On the strategic stability of equilibria, Econometrica 54, $1003-1038$.

Kuhn, H.W. (1953) Extensive games and the problem of information, in Contributions to the Theory of Games 2, 193 - 216, Princeton University Press, Princeton. 
Lemke, C.E., and J.T. Howson Jr. (1964) Equilibrium points of bimatrix games, Journal of the Society for Industrial and Applied Mathematics 12, 413 - 423.

McLennan, A. (1989) Fixed points of contractible valued correspondences, International Journal of Game Theory 18, 175 - 184.

Nash, J.F. (1950) Equilibrium points in n-person games, Proceedings of the National Academy of Science, U.S.A. 36, 48 - 49.

Nash, J.F. (1951) Noncooperative games, Annals of Mathematics 54, 286 - 295.

Papadimitriou, C.H. (1994) On the complexity of the parity argument and other inefficient proofs of existence, Journal of Computer and System Sciences 48 (3), 498 - 532.

Saporiti, A., and F. Tohmé. (2006) Single-crossing, strategic voting and the median choice rule, Social Choice and Welfare 26 (2), 363 - 383.

Savani, R., and B. von Stengel. (2006) Hard-to-solve bimatrix games, Econometrica 74 (2), $397-429$.

\section{Appendix A.}

In this appendix we give a recursive expression of $x_{s}(q, r, T)$, when the distribution applies the upstream mechanism described in section 4. This expression will be used in Appendix B. As noted in section 4, because the distribution center minimizes the shared inventory holding costs, requirements are produced as late as possible. The idea behind the recursive expression is to produce the requirement $r(b, t)$ in the same period as it occurs, namely period $t$, if not it will be produced in period $t-1$, etc. Within a period, suppliers will be addressed in increasing order of their index. Thus, given a feasible ask/bid profile $(q, r)$, the distribution center sets

$$
x_{s}(q, r, T)= \begin{cases}q(s, T) & \text { if } s<s^{T} \\ \sum_{b \in B} r(b, T)-\sum_{\ell=1}^{s-1} q(\ell, T) & \text { if } s=s^{T} \\ 0 & \text { if } s>s^{T}\end{cases}
$$

where $s^{T}$ is defined as the first index $s$ such that

$$
\sum_{b \in B} r(b, T)<\sum_{\ell=1}^{s} q(\ell, T),
$$

or $\infty$ when such an index does not exists. Further, for any $t=T-1, \ldots, 1$ the distribution center sets

$$
x_{s}(q, r, t)= \begin{cases}q(s, t) & \text { if } s<s^{t} \\ \sum_{b \in B} \sum_{\tau=t}^{T} r(b, \tau)-\sum_{\ell \in S} \sum_{\tau=t+1}^{T} x_{\ell}(q, r, \tau)-\sum_{\ell=1}^{s-1} q(\ell, t) & \text { if } s=s^{t} \\ 0 & \text { if } s>s^{t}\end{cases}
$$


where $s^{t}$ is defined as the first index $s$ such that

$$
\sum_{b \in B} \sum_{\tau=t}^{T} r(b, \tau)<\sum_{\ell \in S} \sum_{\tau=t+1}^{T} x_{\ell}(q, r, \tau)+\sum_{\ell=1}^{s} q(\ell, t),
$$

or $\infty$ when such an index does not exists. If $s^{t}<\infty$, we say that $t$ is a critical period and that $s^{t}$ is its critical supplier. Period $t$ is critical if the available capacity plus the scheduled amounts in period $t+1$ through period $T$ is strictly above the total requirements in period $t$ through period $T$. Once we are in a critical period, the corresponding critical supplier is the first one for which the total available capacity of suppliers $1, \ldots, s$ in period $t$ plus the scheduled amounts in period $t+1$ through period $T$ is strictly above the total requirements in period $t$ through period $T$.

Given an ask/bid vector $(q, r)$, the planning horizon decomposes into blocks by using the critical periods. A block is defined as a collection of consecutive periods such that the first period is the only period where the (backward) cumulative requirements are at most the (backward) cumulative capacities. To be precise, $\left[t_{1}, t_{2}\right]$ is a block if and only if

$$
\sum_{b \in B} \sum_{\tau=t}^{t_{2}} r(b, \tau)>\sum_{\ell \in S} \sum_{\tau=t}^{t_{2}} q(\ell, \tau)
$$

for each $t=t_{1}+1, \ldots, t_{2}$, and

$$
\sum_{b \in B} \sum_{\tau=t_{1}}^{t_{2}} r(b, \tau) \leq \sum_{\ell \in S} \sum_{\tau=t_{1}}^{t_{2}} q(\ell, \tau) .
$$

It is easy to see that the critical periods decompose the planning horizon into blocks.

In the following we illustrate these concepts with an example.

Example A.1. Consider a planning horizon consisting of four time periods, i.e. $T=4$, two suppliers and one single buyer. For the suppliers we have

\begin{tabular}{|r|r|r|r|r|}
\hline$t=$ & 1 & 2 & 3 & 4 \\
\hline$c(1, t)=$ & 10 & $\frac{1}{3}$ & $\frac{2}{3}$ & 1 \\
\hline$m(1, t)=$ & 5 & 5 & 5 & 5 \\
\hline$c(2, t)=$ & 15 & 15 & 15 & 15 \\
\hline$m(2, t)=$ & 5 & 5 & 5 & 5 \\
\hline
\end{tabular}

The unit inventory holding costs for the input product faced by the distribution center are equal to 1 , equally paid by the suppliers and the buyer ( $\frac{1}{3}$ per period each).

The requirements of the buyer are equal to $(3,6,6,8)$. Suppose that the first supplier bids $(5,0,0,5)$ and the second supplier his actual capacities, i.e., $(5,5,5,5)$. At period $T$, we have more capacity than the requirements in that period therefore, we will have the first 
critical period and the corresponding critical supplier will be supplier 2. The first supplier will produce his bid, while the second supplier will only produce the needed amount to exactly fulfill the requirements, i.e., 3 units. We can find these figures in the last column of the table below. Given the schedule in the last period, we go one period backwards. The total available capacity in period 3 together with the scheduled amounts in period 4 are not enough to fulfill the total requirements in period 3 and 4 . The same happens in period 2. So the next critical period will be period 1 and the critical supplier will be the first one since his bid together with the scheduled amounts in periods 2 through 4 is enough to fulfill the total requirements from period 1 through period 4 . This ask/bid vector yields a decomposition of the planning horizon into blocks [1,3] and [4].

\begin{tabular}{|r|l|l|l|l|}
\hline$t=$ & 1 & 2 & 3 & 4 \\
\hline$x_{1}(q, r, t)=$ & 5 & 0 & 0 & 5 \\
\hline$x_{2}(q, r, t)=$ & 0 & 5 & 5 & 3 \\
\hline
\end{tabular}

\section{Appendix B.}

In this appendix we show that for the upstream mechanism described in section 4 , where the aim is to minimize the shared inventory holding costs, the values of $M B R_{s}$ are convex. We make use of the recursive expression of $x_{s}(q, r, T)$ derived in Appendix $\mathrm{A}$, and the concepts of block, critical period and critical supplier.

Remark B.1. Let $\left(q^{\prime}, r\right)$ be an element of $M B R_{s}(q, r)$ and let $\left[t_{1}, t_{2}\right]$ be one of the blocks of the decomposition of the planning horizon obtained from $\left(q^{\prime}, r\right)$. If supplier $s$ bids some capacity in the first period of the block $q^{\prime}\left(s, t_{1}\right)>0$, then $x_{\ell}\left(q^{\prime}, r, t_{1}\right)=q^{\prime}\left(\ell, t_{1}\right)$ for all $\ell \neq s$. Otherwise, part of this production could have been done by another supplier, with less production costs for supplier $s$ and the same inventory holding costs.

In the following lemma we show that if we have two ask/bid profiles sharing some of their critical periods, then any convex combination will also share these critical periods.

Lemma B.1. Let $\left(q^{\prime}, r\right)$ and $\left(q^{\prime \prime}, r\right)$ be elements of $M B R_{s}(q, r)$, and $(q(\lambda), r)$ where $q(\lambda):=\lambda q^{\prime}+(1-\lambda) q^{\prime \prime}$ for $0 \leq \lambda \leq 1$. Then, any common critical period for both $\left(q^{\prime}, r\right)$ and $\left(q^{\prime \prime}, r\right)$ is also critical for $(q(\lambda), r)$.

Proof. It is enough to show that

$$
\sum_{\ell \in S} \sum_{\tau=t}^{T} x_{\ell}(q(\lambda), r, \tau) \geq \lambda \sum_{\ell \in S} \sum_{\tau=t}^{T} x_{\ell}\left(q^{\prime}, r, \tau\right)+(1-\lambda) \sum_{\ell \in S} \sum_{\tau=t}^{T} x_{\ell}\left(q^{\prime \prime}, r, \tau\right)
$$

for all $t$. Suppose that these inequalities are true. Take a common critical point for both $\left(q^{\prime}, r\right)$ and $\left(q^{\prime \prime}, r\right)$, say $t$. From the definition of critical point we know that there exists a supplier $s^{t}$ such that the total available capacity of suppliers $1, \ldots, s^{t}$ in period $t$ 
plus the scheduled amounts in period $t+1$ through period $T$ is strictly bigger than the requirements in period $t$ through period $T$, i.e.,

$$
\sum_{\ell \in S} \sum_{\tau=t+1}^{T} x_{\ell}\left(q^{\prime}, r, \tau\right)+\sum_{\ell=1}^{s^{t}} q^{\prime}(\ell, t)>\sum_{b \in B} \sum_{\tau=t}^{T} r(b, \tau)
$$

and

$$
\sum_{\ell \in S} \sum_{\tau=t+1}^{T} x_{\ell}\left(q^{\prime \prime}, r, \tau\right)+\sum_{\ell=1}^{s^{t}} q^{\prime \prime}(\ell, t)>\sum_{b \in B} \sum_{\tau=t}^{T} r(b, \tau) .
$$

Now by taking the corresponding convex combination,

$$
\lambda \sum_{\ell \in S} \sum_{\tau=t+1}^{T} x_{\ell}\left(q^{\prime}, r, \tau\right)+(1-\lambda) \sum_{\ell \in S} \sum_{\tau=t+1}^{T} x_{\ell}\left(q^{\prime \prime}, r, \tau\right)+\sum_{\ell=1}^{s^{t}} q(\lambda)(\ell, t)>\sum_{b \in B} \sum_{\tau=t}^{T} r(b, \tau),
$$

and the result follows from (1).

We can show (1) using backwards induction on $t$. First, we prove that the result is true for $t=T$. We have that

$$
\begin{aligned}
\sum_{\ell \in S} x_{\ell}(q(\lambda), r, T) & =\min \left\{\sum_{\ell \in S} q(\lambda)(\ell, T), \sum_{b \in B} r(b, T)\right\} \\
& =\min \left\{\lambda \sum_{\ell \in S} q^{\prime}(\ell, T)+(1-\lambda) \sum_{\ell \in S} q^{\prime \prime}(\ell, T), \sum_{b \in B} r(b, T)\right\} \\
& \geq \lambda \min \left\{\sum_{\ell \in S} q^{\prime}(\ell, T), \sum_{b \in B} r(b, T)\right\}+(1-\lambda) \min \left\{\sum_{\ell \in S} q^{\prime \prime}(\ell, T), \sum_{b \in B} r(b, T)\right\} \\
& =\lambda \sum_{\ell \in S} x_{\ell}\left(q^{\prime}, r, T\right)+(1-\lambda) \sum_{\ell \in S} x_{\ell}\left(q^{\prime \prime}, r, T\right) .
\end{aligned}
$$

Now suppose that (1) is true for period $t+1$ and we want to prove it for $t$. In that case

$$
\begin{aligned}
& \sum_{\ell \in S} \sum_{\tau=t}^{T} x_{\ell}(q(\lambda), r, \tau)=\min \left\{\sum_{\ell \in S} \sum_{\tau=t+1}^{T} x_{\ell}(q(\lambda), r, \tau)+\sum_{\ell \in S} q(\lambda)(\ell, t), \sum_{b \in B} \sum_{\tau=t}^{T} r(b, t)\right\} \\
& \geq \min \left\{\lambda\left(\sum_{\ell \in S} \sum_{\tau=t+1}^{T} x_{\ell}\left(q^{\prime}, r, \tau\right)+\sum_{\ell \in S} q^{\prime}(\ell, t)\right)\right. \\
&\left.+(1-\lambda)\left(\sum_{\ell \in S} \sum_{\tau=t+1}^{T} x_{\ell}\left(q^{\prime \prime}, r, \tau\right)+\sum_{\ell \in S} q^{\prime \prime}(\ell, t)\right), \sum_{b \in B} \sum_{\tau=t}^{T} r(b, t)\right\}
\end{aligned}
$$

and the proof continues in a similar fashion as above.

Lemma B.2 derives some insight in the plan of a convex combination of two ask/bid profiles $\left(q^{\prime}, r\right),\left(q^{\prime \prime}, r\right) \in M B R_{s}(q, r)$. From this result we can derive that the inventory levels in $(q(\lambda), r)$ are a convex combination of the ones in $\left(q^{\prime}, r\right)$ and $\left(q^{\prime \prime}, r\right)$, which will be used to show that $M B R_{s}(q, r)$ is convex.

Lemma B.2. Let $\left(q^{\prime}, r\right)$ and $\left(q^{\prime \prime}, r\right)$ be elements of $M B R_{s}(q, r)$, and $(q(\lambda), r)$ where $q(\lambda):=\lambda q^{\prime}+(1-\lambda) q^{\prime \prime}$ for $0 \leq \lambda \leq 1$. Then, we have 
1. $x_{\ell}(q(\lambda), r, t)=q(\lambda)(\ell, t)$, for each $\ell \in S$ and $t=2, \ldots, T$,

2. $x_{s}(q(\lambda), r, 1)=q(\lambda)(s, 1)$, and

3. $\sum_{\ell \neq s} x_{\ell}(q(\lambda), r, 1)=\lambda \sum_{\ell \neq s} x_{\ell}\left(q^{\prime}, r, 1\right)+(1-\lambda) \sum_{\ell \neq s} x_{\ell}\left(q^{\prime \prime}, r, 1\right)$.

Proof. Let $\left\{t^{k}\right\}_{k=1}^{K}$ be the joint set of the critical periods of $\left(q^{\prime}, r\right)$ and $\left(q^{\prime \prime}, r\right)$, given in decreasing order.

Because of feasibility we know that $t^{K}=1$. Without loss of generality, we will assume that there is only one period being critical for both $\left(q^{\prime}, r\right)$ and $\left(q^{\prime \prime}, r\right)$, namely $t^{K}=1$. Otherwise, and by using lemma B.1, $(q(\lambda), r)$ will share this critical point as well and the planning horizon can therefore be split using this common critical period. The same proof can be applied to each of the two new planning horizons.

From the definition of a block, Claim 1 follows trivially for $\left(q^{\prime}, r\right)$, i.e. $\lambda=1$, for any period between 2 and the first critical one, and similarly for $\left(q^{\prime \prime}, r\right)$, i.e. $\lambda=0$. For the rest of periods, it is enough to show that for $k=1, \ldots, K-1$

$$
\sum_{b \in B} \sum_{\tau=t^{k}}^{T} r(b, \tau)=\sum_{\ell \in S} \sum_{\tau=t^{k}}^{T} q^{\prime}(\ell, \tau)
$$

when $t^{k}$ is a critical period for $\left(q^{\prime}, r\right)$, and therefore $x_{\ell}\left(q^{\prime}, r, t\right)=q^{\prime}(\ell, t)$ for all $\ell \in S$ and $t \geq t^{k}$, or

$$
\sum_{b \in B} \sum_{\tau=t^{k}}^{T} r(b, \tau)=\sum_{\ell \in S} \sum_{\tau=t^{k}}^{T} q^{\prime \prime}(\ell, \tau)
$$

when $t^{k}$ is a critical period for $\left(q^{\prime \prime}, r\right)$, and therefore $x_{\ell}\left(q^{\prime \prime}, r, t\right)=q^{\prime \prime}(\ell, t)$ for all $\ell \in S$ and $t \geq t^{k}$. Now Claim 1 easily follows for $0<\lambda<1$ from the observation that

$$
\begin{aligned}
\sum_{\ell \in S} \sum_{\tau=2}^{T} x_{\ell}(q(\lambda), r, \tau) & =\sum_{b \in B} \sum_{\tau=2}^{T} r(b, \tau) \\
& =\lambda \sum_{b \in B} \sum_{\tau=2}^{T} r(b, \tau)+(1-\lambda) \sum_{b \in B} \sum_{\tau=2}^{T} r(b, \tau) \\
& =\lambda \sum_{\ell \in S} \sum_{\tau=2}^{T} x_{\ell}\left(q^{\prime}, r, \tau\right)+(1-\lambda) \sum_{\ell \in S} \sum_{\tau=2}^{T} x_{\ell}\left(q^{\prime \prime}, r, \tau\right) \\
& =\lambda \sum_{\ell \in S} \sum_{\tau=2}^{T} q^{\prime}(\ell, \tau)+(1-\lambda) \sum_{\ell \in S} \sum_{\tau=2}^{T} q^{\prime \prime}(\ell, \tau) \\
& =\sum_{\ell \in S} \sum_{\tau=2}^{T} q(\lambda)(\ell, \tau)
\end{aligned}
$$


To show (2) and (3) we will use backwards induction. We will first show the result for $k=1$. Suppose that $t^{1}$ is a critical period of $\left(q^{\prime}, r\right)$ and that $(2)$ does not hold. Then we have

$$
\sum_{b \in B} \sum_{\tau=t^{1}}^{T} r(b, \tau)<\sum_{\ell \in S} \sum_{\tau=t^{1}}^{T} q^{\prime}(\ell, \tau) .
$$

Thus, $q^{\prime}\left(s, t^{1}\right)=0$ by remark B.1. Moreover, because $t^{1}$ is not a critical point of $\left(q^{\prime \prime}, r\right)$

$$
\sum_{\ell \in S} \sum_{\tau=t^{1}}^{T} q^{\prime \prime}(\ell, \tau)<\sum_{b \in B} \sum_{\tau=t^{1}}^{T} r(b, \tau)
$$

from which follows that

$$
\sum_{\tau=t^{1}}^{T} q^{\prime \prime}(s, \tau)<\sum_{\tau=t^{1}}^{T} q^{\prime}(s, \tau) .
$$

We will show that supplier $s$ can reduce the costs associated with $\left(q^{\prime \prime}, r\right)$, contradicting the fact that $\left(q^{\prime \prime}, r\right)$ is a best response for supplier $s$. First observe that the inventory levels in periods $t^{1}-1, \ldots, T$ are strictly positive because these periods belong to the same block. From (4), we know that there exists a period $\tau$ where $m(s, \tau) \geq q^{\prime}(s, \tau)>q^{\prime \prime}(s, \tau)$. (We may observe that $\tau>t^{1}$ because $q^{\prime}\left(s, t^{1}\right)=0$.) In the following we will show that we can decrease the costs associated with $\left(q^{\prime \prime}, r\right)$ by decreasing the inventories levels in periods $t^{1}-1, \ldots, \tau-1$ and producing the corresponding amount in period $\tau$. We notice that the unit production costs in period $\tau$ for supplier $s$ are at most as expensive as the unit inventory costs incurred from period $t^{1}$ up to period $\tau$. This is because in $\left(q^{\prime}, r\right)$ the other suppliers have capacity left during period $t^{1}$. We can construct $\left(q^{\prime \prime \prime}, r\right)$ such that $q^{\prime \prime \prime}(\ell, t)=q^{\prime \prime}(\ell, t)$ for all $(\ell, t) \neq(s, \tau)$ and $q^{\prime \prime \prime}(s, \tau)=q^{\prime \prime}(s, \tau)+\varepsilon$. It is easy to show that $\left(q^{\prime \prime \prime}, r\right) \in \mathcal{D}_{s}(q, r)$ and the costs supplier $s$ are strictly lower in $\left(q^{\prime \prime \prime}, r\right)$ than in $\left(q^{\prime \prime}, r\right)$. This yields the desired reduction on costs.

Now suppose that the result is true for $k-1$ and we want to prove it for $k$. Again and without loss of generality, suppose that $t^{k}$ is a critical point of $\left(q^{\prime}, r\right)$. If (2) does not hold for $t^{k}$, we have

$$
\sum_{b \in B} \sum_{\tau=t^{k}}^{T} r(b, \tau)<\sum_{\ell \in S} \sum_{\tau=t^{k}}^{T} q^{\prime}(\ell, \tau),
$$

where $q^{\prime}\left(s, t^{k}\right)=0$ by using remark B.1, i.e., the rest of the suppliers have capacity left during period $t^{k}$.

Let $t^{\text {low }}$ be the latest critical period of $\left(q^{\prime \prime}, r\right)$ in $t^{1}, \ldots, t^{k-1}$, or $t^{\text {low }}=T+1$ if such a period does not exists. Using (3) for $t^{\text {low }}$ we have that

$$
\sum_{\tau=t^{\mathrm{low}}}^{T} q^{\prime \prime}(s, \tau) \geq \sum_{\tau=t^{\mathrm{low}}}^{T} q^{\prime}(s, \tau) .
$$


Moreover, because $t^{k}$ is not a critical period of $\left(q^{\prime \prime}, r\right)$,

$$
\sum_{\ell \in S} \sum_{\tau=t^{k}}^{T} q^{\prime \prime}(\ell, \tau)<\sum_{b \in B} \sum_{\tau=t^{k}}^{T} r(b, \tau)
$$

and thus

$$
\sum_{\tau=t^{k}}^{T} q^{\prime \prime}(s, \tau)<\sum_{\tau=t^{k}}^{T} q^{\prime}(s, \tau) .
$$

Again, we will show that we can reduce the costs associated with $\left(q^{\prime \prime}, r\right)$. We have that (i) the inventory levels in periods $t^{k}-1, \ldots, t^{\text {low }}-1$ are strictly positive because these periods belong to the same block, (ii) there exists a period $\tau, \tau=t^{k}+1, \ldots, t^{\text {low }}-1$ where $q^{\prime}(s, \tau)>q^{\prime \prime}(s, \tau)$ since (5) and (6) hold and $q^{\prime}\left(s, t^{k}\right)=0$, (iii) the unit production costs in period $\tau$ are at most as expensive as the unit inventory costs incurred from period $t^{k}$ up to period $\tau$ because in $\left(q^{\prime}, r\right)$ the other suppliers have capacity left during period $t^{k}$. In a similar fashion as before, we can show that we can reduce the costs associated with $\left(q^{\prime \prime}, r\right)$, being in contradiction with the fact that $\left(q^{\prime \prime}, r\right)$ is a best response.

In the following we will show Claim 2. We will distinguish three cases.

Case 1: $q^{\prime}(s, 1)$ and $q^{\prime \prime}(s, 1)$ are strictly positive. From remark B.1, we have that $x_{\ell}\left(q^{\prime}, r, 1\right)=q^{\prime}(\ell, 1), x_{\ell}\left(q^{\prime \prime}, r, 1\right)=q^{\prime \prime}(\ell, 1)$, for all $\ell \in S$ and together with Claim 1

$$
\sum_{b \in B} \sum_{\tau=1}^{T} r(b, \tau)=\sum_{\ell \in S} \sum_{\tau=1}^{T} q^{\prime}(\ell, \tau)=\sum_{\ell \in S} \sum_{\tau=1}^{T} q^{\prime \prime}(\ell, \tau) .
$$

Therefore,

$$
\sum_{b \in B} \sum_{\tau=1}^{T} r(b, \tau)=\sum_{\ell \in S} \sum_{\tau=1}^{T} q(\lambda)(\ell, \tau)
$$

and thus, again using Claim $1, x_{\ell}(q(\lambda), r, 1)=q(\lambda)(\ell, 1)$, for all $\ell \in S$. Case 2: $q^{\prime}(s, 1)$. $q^{\prime \prime}(s, 1)=0$ and $q^{\prime}(s, 1)+q^{\prime \prime}(s, 1)>0$. Without loss of generality we analyze the case $q^{\prime}(s, 1)=0$ and $q^{\prime \prime}(s, 1)>0$. From remark B.1, we know that the capacity during period 1 in $\left(q^{\prime \prime}, r\right)$ is fully used. This together with Claim 1 means that

$$
\sum_{b \in B} \sum_{\tau=1}^{T} r(b, \tau)=\sum_{\ell \in S} \sum_{\tau=1}^{T} q^{\prime \prime}(\ell, \tau)
$$

We will show that

$$
\sum_{\ell \in S} \sum_{\tau=1}^{T} q^{\prime}(\ell, \tau)=\sum_{\ell \in S} \sum_{\tau=1}^{T} q^{\prime \prime}(\ell, \tau)
$$

and therefore the capacity in $\left(q^{\prime}, r\right)$ is also fully used. The desired result follows similarly as in Case 1. 
Suppose that (7) is not true, i.e., $\sum_{\ell \in S} \sum_{\tau=1}^{T} q^{\prime \prime}(\ell, \tau)<\sum_{\ell \in S} \sum_{\tau=1}^{T} q^{\prime}(\ell, \tau)$, and thus

$$
\sum_{\tau=1}^{T} q^{\prime \prime}(s, \tau)<\sum_{\tau=1}^{T} q^{\prime}(s, \tau) .
$$

In a similar fashion as above, let $t^{\text {low }}$ be the latest critical period of $\left(q^{\prime \prime}, r\right)$ in $t^{1}, \ldots, t^{K-1}$, or $t^{\text {low }}=T+1$ if such a period does not exists. We have that

$$
\sum_{\tau=t^{\text {low }}}^{T} q^{\prime \prime}(s, \tau) \geq \sum_{\tau=t^{\text {low }}}^{T} q^{\prime}(s, \tau) .
$$

We will show that we can reduce the costs for supplier $s$ associated with $\left(q^{\prime \prime}, r\right)$, which is a contradiction with the fact that this is a best response. We have that (i) the inventory levels during periods $1, \ldots, t^{\text {low }}-1$ are strictly positive because these periods belong to the same block, (ii) part of this inventory is produced by supplier $s$ during period 1 because $q^{\prime \prime}(s, 1)>0$ and the capacity of $\left(q^{\prime \prime}, r\right)$ is fully used, (iii) there exists a period $\tau$, $\tau=2, \ldots, t^{\text {low }}-1$ where $q^{\prime}(s, \tau)>q^{\prime \prime}(s, \tau)$ since (8) and (9) hold and $q^{\prime}(s, 1)=0$, (iv) the unit production costs in period $\tau$ are at most as expensive as the unit inventory costs incurred from period 1 up to period $\tau$ because in $\left(q^{\prime}, r\right)$ the other suppliers have capacity left during period 1 and $x_{s}\left(q^{\prime}, r, \tau\right)=q^{\prime}(s, \tau)>0$. We can construct $\left(q^{\prime \prime \prime}, r\right)$ such that $q^{\prime \prime \prime}(\ell, t)=q^{\prime \prime}(\ell, t)$ for all $(\ell, t) \neq(s, \tau)$ and $q^{\prime \prime \prime}(s, \tau)=q^{\prime \prime}(s, \tau)+\varepsilon$. It is easy to show that $\left(q^{\prime \prime \prime}, r\right) \in \mathcal{D}_{s}(q, r)$ and the costs supplier $s$ are strictly lower in $\left(q^{\prime \prime \prime}, r\right)$ than in $\left(q^{\prime \prime}, r\right)$. This yields the desired reduction on costs.

Case 3: $q^{\prime}(s, 1)=q^{\prime \prime}(s, 1)=0$. We have then $q(\lambda)(s, 1)=0$ and the desired result follows trivially.

It remains to show Claim 3. This easily follows from Claims 1 and 2:

$$
\begin{aligned}
\sum_{b \in B} \sum_{\tau=1}^{T} r(b, \tau)= & \sum_{\ell \in S} \sum_{\tau=1}^{T} x_{\ell}(q(\lambda), r, \tau) \\
= & \sum_{\ell \neq s} x_{\ell}(q(\lambda), r, 1)+x_{s}(q(\lambda), r, 1)+\sum_{\ell \in S} \sum_{\tau=2}^{T} x_{\ell}(q(\lambda), r, \tau) \\
= & \sum_{\ell \neq s} x_{\ell}(q(\lambda), r, 1)+\lambda\left(x_{s}\left(q^{\prime}, r, 1\right)+\sum_{\ell \in S} \sum_{\tau=2}^{T} x_{\ell}\left(q^{\prime}, r, \tau\right)\right) \\
& +(1-\lambda)\left(x_{s}\left(q^{\prime \prime}, r, 1\right)+\sum_{\ell \in S} \sum_{\tau=2}^{T} x_{\ell}\left(q^{\prime \prime}, r, \tau\right)\right) \\
= & \sum_{\ell \neq s} x_{\ell}(q(\lambda), r, 1)+\lambda\left(\sum_{b \in B} \sum_{\tau=1}^{T} r(b, \tau)-\sum_{\ell \neq s} x_{\ell}\left(q^{\prime}, r, 1\right)\right) \\
& +(1-\lambda)\left(\sum_{b \in B} \sum_{\tau=1}^{T} r(b, \tau)-\sum_{\ell \neq s} x_{\ell}\left(q^{\prime \prime}, r, 1\right)\right)
\end{aligned}
$$


and the desired equality follows.

Now we can show that $M B R_{s}(q, r)$ is convex.

Lemma B.3. The values of the correspondence $M B R_{s}$ are convex.

Proof. Take two elements $\left(q^{\prime}, r\right)$ and $\left(q^{\prime \prime}, r\right)$ in $M B R_{s}(q, r)$. Write $q(\lambda):=\lambda q^{\prime}+(1-\lambda) q^{\prime \prime}$, for $0 \leq \lambda \leq 1$. We have to prove that $(q(\lambda), r)$ is an element of $M B R_{s}(q, r)$. We check (i) feasibility, (ii) best response and (iii) $x_{s}(q(\lambda), r, t)=q(\lambda)(s, t)$. To show (i), i.e. $(q(\lambda), r)$ is an element of $\mathcal{D}_{s}(q, r)$, simply observe that this is a convex set. Claim (iii) has been proved in lemma B.2. It remains to show that $(q(\lambda), r)$ is an element of $B R_{s}(q, r)$.

Lemma B.2 ensures that the inventory levels in $(q(\lambda), r)$ are a convex combination of the ones in $\left(q^{\prime}, r\right)$ and $\left(q^{\prime \prime}, r\right)$, i.e.,

$$
G(q(\lambda), r, t)=\lambda G\left(q^{\prime}, r, t\right)+(1-\lambda) G\left(q^{\prime \prime}, r, t\right)
$$

Therefore,

$$
\begin{aligned}
V_{s}(q(\lambda), r) & :=\sum_{t=1}^{T} c(s, t) x_{s}(q(\lambda), r, t)+\frac{1}{|B|+|S|} \sum_{t=1}^{T} g(t) G(q(\lambda), r, t) \\
& =\left[\lambda \sum_{t=1}^{T} c(s, t) x_{s}\left(q^{\prime}, r, t\right)+\frac{1}{|B|+|S|} \sum_{t=1}^{T} g(t) G\left(q^{\prime}, r, t\right)\right] \\
& +(1-\lambda)\left[\sum_{t=1}^{T} c(s, t) x_{s}\left(q^{\prime \prime}, r, t\right)+\frac{1}{|B|+|S|} \sum_{t=1}^{T} g(t) G\left(q^{\prime \prime}, r, t\right)\right] \\
& =\lambda V_{s}\left(q^{\prime}, r\right)+(1-\lambda) V_{s}\left(q^{\prime \prime}, r\right),
\end{aligned}
$$

and the desired result follows. 